TIFR-TH-97/39

hep-th/9707132

\title{
Probing Type I' String Theory Using D0 and D4-Branes
}

\author{
Justin R. David*, Avinash Dhar* and Gautam Mandal* \\ Theoretical Physics Group, Tata Institute of Fundamental Research \\ Homi Bhabha Road, Mumbai 400 005, INDIA.
}

\begin{abstract}
We analyse the velocity-dependent potentials seen by D0 and D4-brane probes moving in Type $I^{\prime}$ background for head-on scattering off the fixed planes. We find that at short distances (compared to string length) the D0-brane probe has a nontrivial moduli space metric, in agreement with the prediction of Type I' matrix model; however, at large distances it is modified by massive open strings to a flat metric, which is consistent with the spacetime equations of motion of Type I' theory. We discuss the implication of this result for the matrix model proposal for M-theory. We also find that the nontrivial metric at short distances in the moduli space action of the D0-brane probe is reflected in the coefficient of the higher dimensional $v^{4}$ term in the D4-brane probe action.
\end{abstract}

\footnotetext{
* e-mail: justin, adhar, mandal, @theory.tifr.res.in
} 


\section{Introduction}

The matrix theory conjecture [1] is a remarkable attempt to provide a microscopic (nonperturbative) description of M-theory [2, 3], which is believed to provide a unified description of all the known 10-dimensional superstring theories. While evidence in favour of this conjecture has been rapidly mounting 田, 5, 6, 7], some serious problems have also surfaced in implementing the usual prescriptions for compactifications of M-theory within the matrix theory framework 1 . Notable among these is the observation by Douglas, Ooguri and Shenker [9] that for M-theory compactifications on curved manifolds a truncation to a finite number of open string degrees of freedom cannot reproduce supergravity results. The example of K3 compactification discussed by these authors has only eight supersymmetries. A similar problem has also been noted [10] recently for possible matrix theory descriptions of M-theory on other nontrivial backgrounds which preserve only eight supersymmetries.

In this note we wish to point out that the matrix quantum mechanics model constructed for Type I' string theory [11, 12, 13] also suffers from this problem. Type I' string theory is S-dual to $E 8 \times E 8$ heterotic string theory and is obtained by compactifying M-theory on $S^{1} \times\left(S^{1} / Z_{2}\right)$ or Type IIA on $S^{1} / Z^{2}$ [14, 15]. Vanishing of total RR charge requires, in this compactification, the presence of 16 D8-branes. The matrix quantum mechanics model has eight superymmetries and belongs to the general class of supersymmetric quantum mechanical models studied recently by Banks, Seiberg and Silverstein [16]. These authors have pointed out that in such models the reduced supersymmetry allows for nonzero loop corrections to the moduli space metric. In particular, in the Type I' matrix model, D0-branes perceive a nontrivial metric, first computed in [11]. This immediately raises a puzzle, as pointed out in [16], since the spacetime equations of motion of Type $\mathrm{I}^{\prime}$ theory lead to a flat metric and a constant dilaton when the 8 D8-branes are placed at each of the two orientifold planes [17]. A constant dilaton [18] and a flat metric are indeed what is perceived on a D4-brane probe. To preserve a probe-independent notion of an underlying spacetime metric at scales larger than the string scale one would have expected the D0-brane also to perceive a flat metric. Our calculations show that this is indeed the case. This then implies that the proposed matrix model of Type $\mathrm{I}^{\prime}$ string theory cannot reproduce the supergravity results of the latter.

We perform and analyse a full string theoretic computation of the velocity-dependent potentials seen respectively by D0-brane and D4-brane probes for head-on scattering off the fixed planes. We find that at large distances (compared with the string scale) the potentials seen by both the probes vanish when 8 D8-branes are on top of the orientifold plane. This is consistent with an underlying flat spacetime and constant dilaton. At short distances, however, the D0-brane has a nontrivial moduli space metric. It is clear from the computation that massive open string excitations contribute to convert the nontrivial metric seen at short distances to a flat metric at large distances. Thus, there is no decoupling of massive open string degrees of freedom as required by the matrix theory conjecture.

The fact that at short distances the D0-brane probe sees a very different moduli space

\footnotetext{
${ }^{1}$ See [8] for a recent summary of developments in this area.
} 
metric than the one seen by the D4-brane probe might seem to preclude the possibility of a probe-independent characterization of an underlying spacetime at short distances. However, our analysis of the velocity-dependent potential seen by the D4-brane probe shows that the metric on the moduli space of the D0-brane probe is reflected in the coefficient of the $v^{4}$ term in the moduli space action of the D4-brane probe. Thus, a probe-independent characterization of an underlying spacetime might still exist in which the metric, dilaton etc. simply couple differently to different probes [16].

The organization of this note is as follows. In section 2 we study the head-on scattering of D0-branes and D4-branes respectively against one of the orientifold fixed planes together with 8 D8-branes of Type $\mathrm{I}^{\prime}$ theory. We compute the velocity-dependent potential seen by a D0-brane in this background and examine its behaviour at large and small distances compared to the string length. At large distances the dominant contribution is from massless closed strings. With 8 D8-branes at each of the two orientifold fixed planes, the entire contribution from the massless closed strings cancels. At short distances the dominant contribution is from the massless open string sector. For the above configuration of D8-branes the potential starts at the $v^{2}$ term and gives rise to a nontrivial moduli space metric for D0-branes. We discuss the large $N$ limit and implications of these results for the matrix model conjecture for M-theory.

In section 3 we repeat the above calculation for a D4-brane probe. At large distances we again find that the contribution from the massless closed strings to the velocity-dependent potential vanishes. At short distances, however, we find a nontrivial potential. The $v^{2}$ term vanishes, giving rise to a flat moduli space metric for the D4-branes, but there is a nonvanishing $v^{4}$ term. Interestingly, the coefficient of this term is essentially the same as that of the $v^{2}$ term in the moduli space action of the D0-brane probe (both are proportional to $1 / r^{3}$ where $r$ is the distance from the orientifold plane). We discuss the implications of this for a possible probe-independent characterization of an underlying spacetime physics.

Section 4 contains a discussion of the results and some concluding remarks.

\section{Scattering of D0-branes}

Type $\mathrm{I}^{\prime}$ theory is obtained from Type IIA by compatifying the $X^{9}$ direction on a circle and performing a world sheet parity $\Omega$ projection and a spacetime parity $P^{9}$ projection along the $X^{9}$ direction. The $P^{9}$ projection leads to fixed 8-planes located at 0 and $\pi R_{I^{\prime}}$ along the $X^{9}$ direction, which we call the orientifold plane or the $\Omega 8$-plane. Each of these fixed planes carries -8 units of RR charge. Thus for charge neutrality in the compact direction one adds 16 D8branes. Our main focus will be on the configuration with 8 D8-branes at each orientifold plane. We will probe this configuration with a D0-brane moving along the $X^{9}$ direction, later on to be generalized to $N$ D0-branes. In the closed string channel the contribution to the one loop vacuum amplitude from one of the fixed planes, say $X^{9}=0$, is the sum of the cylinder diagrams between the D0-brane probe and the 8 D8-branes and the cross cap between the probe and the orientifold plane. We do this computation using the boundary state formalism for scattering of moving D-branes [19, 20]. 


\section{$2.1 \quad \mathrm{D} 0-\Omega 8$ interaction}

The result for the one-loop vacuum amplitude describing the static $\Omega 8$-plane and the D0-brane moving in the $X^{9}$ direction is

$$
\begin{array}{r}
\mathcal{A}_{D 0-\Omega 8}=4 \int_{0}^{\infty} \frac{d \tau}{\pi} \frac{\Theta_{1}^{\prime}(0 \mid i \tau+1 / 2)}{i \Theta_{1}(-i \epsilon \mid i \tau+1 / 2)} \times\left\{\left[\frac{\Theta_{3}(0 \mid i \tau+1 / 2)}{\Theta_{2}(0 \mid i \tau+1 / 2)}\right]^{4} \frac{\Theta_{4}(-i \epsilon \mid i \tau+1 / 2)}{\Theta_{4}(0 \mid i \tau+1 / 2)}\right. \\
\left.-\left[\frac{\Theta_{4}(0 \mid i \tau+1 / 2)}{\Theta_{2}(0 \mid i \tau+1 / 2)}\right]^{4} \frac{\Theta_{3}(-i \epsilon \mid i \tau+1 / 2)}{\Theta_{3}(0 \mid i \tau+1 / 2)}\right\}+\infty \times 4 \int_{0}^{\infty} d \tau
\end{array}
$$

This result agrees with the calculation reported recently in [21]. In the above equation $\pi \epsilon=$ $\tanh ^{-1} v$ where $v$ is the velocity of the D0-brane along the $X^{9}$ direction. The infinity in the ramond sector of the amplitude is due to the contribution of the superghost ground state, and is peculiar to the case in which the number of Neumann-Dirichlet boundary conditions is 8 [21]. The divergent contribution need not worry us as it cancels in the physical amplitude which is the sum of scatterings from the 8 D8-branes and the orientifold plane. The sign in front of the divergent term is fixed to be ' + ' as the D0-brane and the $\Omega 8$-plane have opposite signs of $\mathrm{RR}$ charge. Note that there is no impact parameter for this head-on scattering problem. The velocity-dependant potential $\mathcal{V}$ can be calculated from the above amplitude using the procedure of [23, 24]. It is given by,

$$
\begin{array}{r}
\mathcal{V}_{D 0-\Omega 8}=\frac{4 \sinh \pi \epsilon}{\sqrt{2 \pi^{2} \alpha^{\prime}}} \int_{0}^{\infty} \frac{d \tau e^{-\frac{r^{2}}{2 \pi \tau \alpha^{\prime}}}}{\pi \sqrt{\tau}} \frac{\Theta_{1}^{\prime}(0 \mid i \tau+1 / 2)}{i \Theta_{1}(-i \epsilon \mid i \tau+1 / 2)} \times\left\{\left[\frac{\Theta_{3}(0 \mid i \tau+1 / 2)}{\Theta_{2}(0 \mid i \tau+1 / 2)}\right]^{4} \frac{\Theta_{4}(-i \epsilon \mid i \tau+1 / 2)}{\Theta_{4}(0 \mid i \tau+1 / 2)}\right. \\
\left.-\left[\frac{\Theta_{4}(0 \mid i \tau+1 / 2)}{\Theta_{2}(0 \mid i \tau+1 / 2)}\right]^{4} \frac{\Theta_{3}(-i \epsilon \mid i \tau+1 / 2)}{\Theta_{3}(0 \mid i \tau+1 / 2)}\right\}+\infty \times \frac{4 \sinh \pi \epsilon}{\sqrt{2 \pi^{2} \alpha^{\prime}}} \int_{0}^{\infty} \frac{d \tau}{\sqrt{\tau}} e^{-\frac{r^{2}}{2 \pi \tau \alpha^{\prime}}}
\end{array}
$$

where $r^{2}=\left(X_{(0)}^{9}+X^{0} \sinh \pi \epsilon\right)^{2}$. At large distances $\left(r>>\sqrt{\alpha^{\prime}}\right)$, the amplitude is dominated by exchange of massless closed strings. In this regime the leading term in the potential is obtained by expanding the $\Theta$ functions in powers of $q=e^{-\pi \tau}$ and retaining only the zeroth order term. We get,

$$
\mathcal{V}_{D 0-\Omega 8}=\frac{r(\cosh 2 \pi \epsilon-5)}{\pi \alpha^{\prime}}
$$

We have ignored the divergent term keeping in mind that it will cancel a similar divergent term from the 8 D8-branes. At short distances $\left(r<<\sqrt{\alpha^{\prime}}\right)$ massless open strings dominate the amplitude. To extract their contribution it is convenient to perform a world sheet duality transformation and convert the cross cap to a mobius strip. This is easily done by substituting $\tau=1 / 4 t$ and using the modular properties of the $\Theta$ functions. The modular parameter of the $\Theta$ functions changes from $i \tau+1 / 2$ to $i / 4 t+1 / 2$. The corresponding modular transformation is

$$
\left(\begin{array}{ll}
1 & -1 \\
2 & -1
\end{array}\right)
$$


This can be obtained by the following sequence of $\mathrm{S}$ and $\mathrm{T}$ transformations,

$$
T S T^{2} S
$$

These lead to the list of formulae given below.

$$
\begin{aligned}
& \Theta_{1}(z \mid i / 4 t+1 / 2)=\exp (i 3 \pi / 4)(-2 i t)^{1 / 2} \exp \left(-4 \pi z^{2} t\right) \Theta_{1}(2 i t z \mid i t+1 / 2) \\
& \Theta_{1}^{\prime}(0 \mid i / 4 t+1 / 2)=\exp (i 3 \pi / 4)(2 i t)(-2 i t)^{1 / 2} \Theta_{1}^{\prime}(0 \mid i t+1 / 2) \\
& \Theta_{2}(z \mid i / 4 t+1 / 2)=\exp (i \pi / 4)(-2 i t)^{1 / 2} \exp \left(-4 \pi z^{2} t\right) \Theta_{2}(2 i t z \mid i t+1 / 2) \\
& \Theta_{3}(z \mid i / 4 t+1 / 2)=\exp (i \pi / 2)(-2 i t)^{1 / 2} \exp \left(-4 \pi z^{2} t\right) \Theta_{4}(2 i t z \mid i t+1 / 2) \\
& \Theta_{4}(z \mid i / 4 t+1 / 2)=(-2 i t)^{1 / 2} \exp \left(-4 \pi z^{2} t\right) \Theta_{3}(2 i t z \mid i t+1 / 2)
\end{aligned}
$$

Using these transformations we obtain the following expression for the potential,

$$
\begin{array}{r}
\mathcal{V}_{D 0-\Omega 8}=\frac{4 \sinh \pi \epsilon}{\sqrt{2 \pi^{2} \alpha^{\prime}}} \int_{0}^{\infty} \frac{d t e^{-\frac{2 r^{2} t}{\pi \alpha^{\prime}}}}{\pi \sqrt{t}} \frac{\Theta_{1}^{\prime}(0 \mid i t+1 / 2)}{\Theta_{1}(2 \epsilon t \mid i t+1 / 2)} \times\left\{\left[\frac{\Theta_{3}(0 \mid i t+1 / 2)}{\Theta_{2}(0 \mid i t+1 / 2)}\right]^{4} \frac{\Theta_{4}(\epsilon t \mid i t+1 / 2)}{\Theta_{4}(0 \mid i t+1 / 2)}\right. \\
\left.-\left[\frac{\Theta_{4}(0 \mid i t+1 / 2)}{\Theta_{2}(0 \mid i t+1 / 2)}\right]^{4} \frac{\Theta_{3}(2 \epsilon t \mid i t+1 / 2)}{\Theta_{3}(0 \mid i t+1 / 2)}\right\}+\infty \times \frac{2 \sinh \pi \epsilon}{\sqrt{2 \pi^{2} \alpha^{\prime}}} \int_{0}^{\infty} \frac{d t}{t^{3 / 2}} e^{-\frac{2 r^{2} t}{\pi \alpha^{\prime}}}
\end{array}
$$

It is interesting to note an effective doubling of the distance $r$ and the rapidity parameter $\pi \epsilon$ in the above expression. Thus, even though (7) is obtained from a closed string calculation by a world sheet duality, the picture of an open string stretching between the D0-brane and its image (reflected in the orientifold plane) automatically emerges. The contribution of massless open strings to the potential is then given by

$$
\mathcal{V}_{D 0-\Omega 8}=\frac{2 \sinh \pi \epsilon}{\sqrt{2 \pi^{2} \alpha^{\prime}}} \int_{0}^{\infty} d t \frac{e^{\frac{-r^{2} t}{2 \pi \alpha^{\prime}}}}{\sqrt{t} \sin (\pi \epsilon t / 2)}\left(1-\frac{\cos \pi \epsilon t-1}{4}\right)
$$

In obtaining the above expression we have made the change of variable $t \rightarrow t / 4$. In the region $\sqrt{\alpha^{\prime}}>>r>\sqrt{2 \pi^{2} \alpha^{\prime} \epsilon}$ we can perform a low velocity expansion of the potential. This gives

$$
\mathcal{V}_{D 0-\Omega 8}=-\left(1+\frac{v^{2}}{6}\right) \frac{4 r}{\pi \alpha^{\prime}}+\frac{2 v^{2} \pi \alpha^{\prime}}{3 r^{3}}+O\left(v^{4}\right)
$$

where we have used $\pi \epsilon=v+o\left(v^{3}\right)$. As a check on our calculations we compare (3) and (9) and note that the coefficient of the leading term in the velocity expansion, that is the term linear in $r$, matches. This is as expected since the difference in the dimension of the probe and the target is $0 \bmod 4$ [24].

\subsection{D0-D8 interaction}

Let us now find the potential felt by a D0-brane as it scatters off the D8-branes at the fixed plane. To do this we compute the cylinder diagram between the D8-brane and the D0-brane. 
We find the potential to be

$$
\begin{array}{r}
\mathcal{V}_{D 0-D 8}=\frac{\sinh \pi \epsilon}{\sqrt{2 \pi^{2} \alpha^{\prime}}} \int_{0}^{\infty} d \tau \frac{e^{\frac{-r^{2}}{2 \pi \alpha^{\prime} \tau}}}{4 \sqrt{\tau}} \frac{1}{f_{2}^{8}\left(e^{-\pi \tau}\right)} \frac{\Theta_{1}^{\prime}(0 \mid i \tau)}{i \pi \Theta_{1}(-i \epsilon \mid i \tau)} \times \\
\left\{f_{4}^{8}\left(e^{-\pi \tau}\right) \frac{\Theta_{3}(-i \epsilon \mid i \tau)}{\Theta_{3}(0 \mid i \tau)}-f_{3}^{8}\left(e^{-\pi \tau}\right) \frac{\Theta_{4}(-i \epsilon \mid i \tau)}{\Theta_{4}(0 \mid i \tau)}\right\}-\infty \times \frac{\sinh \pi \epsilon}{\sqrt{2 \pi^{2} \alpha^{\prime}}} \int_{0}^{\infty} d \tau \frac{e^{-\frac{r^{2}}{2 \pi \alpha^{\prime} \tau}}}{4 \sqrt{\tau}}
\end{array}
$$

which agrees with the calculations recently reported in [21, 22]. In the above expression we have fixed the sign in front of the contribution from the Ramond sector to be '-' as the D0-brane and the D8-brane have the same sign of the RR charge. The $f$ 's are as defined in 25. We extract the contribution of the massless closed strings relevant at long distances (neglecting the infinity as explained before) to be

$$
\mathcal{V}_{D 0-D 8}=\frac{r(5-\cosh 2 \pi \epsilon)}{16 \pi \alpha^{\prime}}
$$

To find the contribution from the 8 D8-branes at the fixed plane we must multiply $\mathcal{V}_{D 0-D 8}$ by 16 . The factor of 16 can be seen from two points of view. The 8 D8-branes sitting at the orientifold plane enhance the spacetime gauge group to $S O(16)$, so the Chan-Paton factors are in the fundamental of $S O(16) \times U(1)$. Tracing over these gives a factor of 16 . If one thinks in terms of the open string picture, we have to add the contribution of the 8 D8-branes and their images. This gives the factor of 16 .

We note that, as promised earlier, in the sum

$$
\mathcal{V}_{D 0-\Omega 8}+16 \mathcal{V}_{D 0-D 8}
$$

the infinities cancel leaving behind a finite answer for the physical configuration. We also note that this sum vanishes in the massless closed string sector. Thus at long distances we have a flat spacetime and constant dilaton background from the point of view of the D0-brane probe.

The short distance behaviour of the potential in (10) is found by substituting $\tau=1 / t$ and using the modular properties of the $\Theta$ functions and the $f$ 's. We get the following result

$$
\begin{array}{r}
\mathcal{V}_{D 0-D 8}=\frac{\sinh \pi \epsilon}{\sqrt{2 \pi^{2} \alpha^{\prime}}} \int_{0}^{\infty} d t \frac{e^{\frac{-r^{2} t}{2 \pi \alpha^{\prime}}}}{4 \pi \sqrt{t}} \frac{1}{f_{4}^{8}\left(e^{-\pi t}\right)} \frac{\Theta_{1}^{\prime}(0 \mid i t)}{\Theta_{1}(\epsilon t \mid i t)} \times \\
\left\{f_{2}^{8}\left(e^{-\pi t}\right) \frac{\Theta_{3}(\epsilon t \mid i t)}{\Theta_{3}(0 \mid i t)}-f_{3}^{8}\left(e^{-\pi t}\right) \frac{\Theta_{2}(\epsilon t \mid i t)}{\Theta_{2}(0 \mid i t)}\right\}-\infty \times \frac{\sinh \pi \epsilon}{\sqrt{2 \pi^{2} \alpha^{\prime}}} \int_{0}^{\infty} \frac{e^{-\frac{r^{2} t}{2 \pi \alpha^{\prime}}}}{4 t^{3 / 2}}
\end{array}
$$

The contribution of the massless open string modes is

$$
\mathcal{V}_{D 0-D 8}=-\frac{\sinh \pi \epsilon}{\sqrt{2 \pi^{2} \alpha^{\prime}}} \int_{0}^{\infty} d t \frac{e^{\frac{-r^{2} t}{2 \pi \alpha^{\prime}}}}{4 \sqrt{t}} \cot \pi \epsilon t
$$

and its velocity expansion is,

$$
\mathcal{V}_{D 0-D 8}=\left(1+\frac{v^{2}}{6}\right) \frac{r}{4 \pi \alpha^{\prime}}+\frac{v^{2} \pi \alpha^{\prime}}{12 r^{3}}+O\left(v^{4}\right)
$$


Finally, for the physical sum, we get

$$
\mathcal{V}_{D 0-\Omega 8}+16 \mathcal{V}_{D 0-D 8}=\frac{2 v^{2} \pi \alpha^{\prime}}{r^{3}}+O\left(v^{4}\right)
$$

We see that, as expected, the linear term cancels and we are left with a effective potential which starts off at order $v^{2}$. This implies that there is a metric on the moduli space of the D0-brane. One can write this metric as

$$
d s^{2}=\frac{1}{2 \lambda_{I^{\prime}}}\left(1+\frac{4 \pi \alpha^{\prime 3 / 2} \lambda_{I^{\prime}}}{r^{3}}\right) d r^{2}
$$

where $\lambda_{I^{\prime}}$ is the string coupling.

\subsection{Large $N$ limit}

We would now like to extend the above results to the case of scattering of a bound state of $N$ D0-branes off the fixed plane. To the lowest order in the string coupling the potentials for this case are simply a factor of $N$ larger than those given by (2) and (8). This is simple to understand for the D0-D8 scattering. For D0- $\Omega 8$ scattering this factor of $N$ arises as follows. To compute the mobius strip one inserts the world sheet projection operator $\Omega$ and the space time projection operator $P^{9}$ in the trace over open string states. The eigenvalue of $\Omega P^{9}$ on symmetric (antisymmetric) Chan-Paton factors is $+1(-1)$. Since the Hamiltonian of the open string does not depend on the Chan-Paton factors, the multiplicities come as a prefactor in the one-loop vacuum amplitude, giving an overall factor $N(N+1) / 2-N(N-1) / 2=N$ in the amplitude. Thus to lowest order in string coupling the short distance potential for the scattering of a bound state of $\mathrm{N}$ D0-branes against the orientifold with 8 D8-branes is

$$
\mathcal{V}_{D 0-\Omega 8}+16 \mathcal{V}_{D 0-D 8}=\frac{N 2 v^{2} \pi \alpha^{\prime}}{r^{3}}+O\left(v^{4}\right)
$$

The nontrivial correction away from flat metric in (18) does not disappear in the large $N$ limit even when we take into account the $N$ scalings of space and time variables [1, 10]. In higher orders of string coupling, ordinary large $N$ counting suggests that the lowest order result could get modified by a factor which is a function of the ratio $N / r^{3}$. Thus the above conclusion cannot be modified by higher order perturbative corrections. It is clear from our calculations that the source of the problem is that the massive open string modes do not decouple at large distances. The implication for the matrix model proposal for Type $\mathrm{I}^{\prime}$ theory [11, 12, 13] is that it does not give the correct description of the eleven dimensional physics of M-theory.

\section{Scattering of D4-branes}

In order to discuss the probe-dependence of the above results, we now repeat the above calculations with a D4-brane probe. The choice of a D4-brane probe is dictated by the fact that it is the only other probe which obeys the condition that the difference of dimensions of the probe and the target is a multiple of four. 


\subsection{D4- $\Omega 8$ interaction}

We perform similar computations as in the D0-brane case. The velocity-dependent potential seen by the D4-brane probe due to the orientifold is

$$
\begin{array}{r}
\mathcal{V}_{D 4-\Omega 8}=\frac{2^{4}(2 \pi)^{2} \sinh \pi \epsilon V_{4}}{\sqrt{2 \pi^{2} \alpha^{\prime}}} \int_{0}^{\infty} \frac{d \tau}{\sqrt{\tau}} \frac{e^{-\frac{r^{2}}{2 \pi \alpha^{\prime} \tau}}}{\left(8 \pi^{2} \alpha^{\prime}\right)^{2}} \frac{\Theta_{1}^{\prime}(0 \mid i \tau+1 / 2)}{i \pi \Theta_{1}(-i \epsilon \mid i \tau+1 / 2)} \times \\
\left\{\frac{\Theta_{3}(0 \mid i \tau+1 / 2) \Theta_{4}(0 \mid i \tau+1 / 2)}{\Theta_{1}^{\prime}(0 \mid i \tau+1 / 2) \Theta_{2}(0 \mid i \tau+1 / 2)}\right\}^{2} \times\left\{\frac{\Theta_{4}(-i \epsilon \mid i \tau+1 / 2)}{\Theta_{4}(0 \mid i \tau+1 / 2)}-\frac{\Theta_{3}(-i \epsilon \mid i \tau+1 / 2)}{\Theta_{3}(0 \mid i \tau+1 / 2}\right\}
\end{array}
$$

In the above expression $V_{4}$ is the four volume of the D4-brane. At large distances the potential is

$$
\mathcal{V}_{D 4-\Omega 8}=\frac{4 V_{4}}{\left(8 \pi^{2} \alpha^{\prime}\right)^{2}} \frac{r}{\pi \alpha^{\prime}}(\cosh 2 \pi \epsilon-1)
$$

In the variable $t=1 / 4 \tau$ the potential is given by

$$
\begin{gathered}
\mathcal{V}_{D 4-\Omega 8}=\frac{2^{4}(2 \pi)^{2} \sinh \pi \epsilon V_{4}}{\sqrt{2 \pi^{2} \alpha^{\prime}}} \int_{0}^{\infty} \frac{d t}{16 \sqrt{t}} \frac{e^{\frac{-2 r^{2} t}{\pi \alpha^{\prime}}}}{\left(8 \pi^{2} \alpha^{\prime} t\right)^{2}} \frac{\Theta_{1}^{\prime}(0 \mid i t+1 / 2)}{\pi \Theta_{1}(2 \epsilon t \mid i t+1 / 2)} \times \\
\left\{\frac{\Theta_{4}(0 \mid i t+1 / 2) \Theta_{3}(0 \mid i t+1 / 2)}{\Theta_{1}^{\prime}(0 \mid i t+1 / 2) \Theta_{2}(0 \mid i t+1 / 2)}\right\}^{2} \times\left\{\frac{\Theta_{3}(2 \epsilon t \mid i t+1 / 2)}{\Theta_{3}(0 \mid i t+1 / 2)}-\frac{\Theta_{4}(2 \epsilon t \mid i t+1 / 2)}{\Theta_{4}(0 \mid i t+1 / 2}\right\}
\end{gathered}
$$

This gives the dominant contribution to the potential at short distances to be

$$
\mathcal{V}_{D 4-\Omega 8}=\frac{2^{4} \sinh \pi \epsilon V_{4}}{\sqrt{2 \pi^{2} \alpha^{\prime}}} \int_{0}^{\infty} \frac{d t}{2 \sqrt{t}} \frac{e^{-\frac{r^{2} t}{2 \pi \alpha^{\prime}}}}{\left(8 \pi^{2} \alpha^{\prime} t\right)^{2}} \frac{\cos \pi \epsilon t-1}{\sin \pi \epsilon t / 2}
$$

Note that in the above expression we have changed the variable of integration from $t \rightarrow t / 4$. Performing a velocity expansion, we get

$$
\mathcal{V}_{D 4-\Omega 8}=\frac{2^{4} V_{4}}{\left(8 \pi^{2} \alpha^{\prime}\right)^{2}} \frac{v^{2}}{2}\left(\frac{r\left(1-v^{2} / 2\right)}{\pi \alpha^{\prime}}+\frac{v^{2}}{24} \frac{\pi \alpha^{\prime}}{r^{3}}\right)+O\left(v^{6}\right)
$$

\subsection{D4-D8 interaction}

The same calculations are now repeated for the D8-brane case. The velocity dependent potential seen by the D4-brane is

$$
\begin{gathered}
\mathcal{V}_{D 4-D 8}=\frac{V_{4} \sinh \pi \epsilon}{\sqrt{2 \pi^{2} \alpha^{\prime}}} \int_{0}^{\infty} \frac{d \tau}{4 \sqrt{\tau}} \frac{e^{\frac{-r^{2}}{2 \pi \alpha^{\prime} \tau}}}{\left(8 \pi^{2} \alpha^{\prime}\right)^{2}} \frac{\Theta_{1}^{\prime}(0 \mid i \tau)}{i \pi \Theta_{1}(-i \epsilon \mid i \tau)} \times \\
\left\{\frac{f_{3}\left(e^{-\pi \tau}\right) f_{4}\left(e^{-\pi \tau}\right)}{f_{1}\left(e^{-\pi \tau}\right) f_{2}\left(e^{-\pi \tau}\right)}\right\}^{4} \times\left\{\frac{\Theta_{3}(-i \epsilon \mid i \tau)}{\Theta_{3}(0 \mid i \tau)}-\frac{\Theta_{4}(-i \epsilon \mid i \tau)}{\Theta_{4}(0 \mid i \tau)}\right\}
\end{gathered}
$$

So at large distances we get

$$
\mathcal{V}_{D 4-D 8}=-\frac{r}{\pi \alpha^{\prime}} \frac{V_{4}}{\left(8 \pi^{2} \alpha^{\prime}\right)^{2}} \frac{\cosh 2 \pi \epsilon-1}{4}
$$


Again we note that the sum

$$
\mathcal{V}_{D 4-\Omega 8}+16 \mathcal{V}_{D 4-D 8}=0
$$

At large distances this is consistent with the zero brane result. This confirms that at large distances we have a probe-independent description in terms of a flat spacetime and constant dilaton background.

To extract the short distance behaviour of the potential we make the substitution $\tau=1 / t$ in (24). This gives

$$
\begin{array}{r}
\mathcal{V}_{D 4-D 8}=\frac{V_{4} \sinh \pi \epsilon}{\sqrt{2 \pi^{2} \alpha^{\prime}}} \int_{0}^{\infty} \frac{d t}{4 \sqrt{t}} \frac{e^{\frac{-r^{2} t}{2 \pi \alpha^{\prime}}}}{\left(8 \pi^{2} \alpha^{\prime} t\right)^{2}} \frac{\Theta_{1}^{\prime}(0 \mid i t)}{\pi \Theta_{1}(i \epsilon t \mid i t)} \times \\
\left\{\frac{f_{3}\left(e^{-\pi t}\right) f_{2}\left(e^{-\pi t}\right)}{f_{1}\left(e^{-\pi \tau}\right) f_{4}\left(e^{-\pi \tau}\right)}\right\}^{4} \times\left\{\frac{\Theta_{3}(\epsilon t \mid i t)}{\Theta_{3}(0 \mid i t)}-\frac{\Theta_{2}(\epsilon t \mid i t)}{\Theta_{2}(0 \mid i t)}\right\}
\end{array}
$$

This result has also been reported recently in [22]. The dominant contribution at short distances is

$$
\mathcal{V}_{D 4-D 8}=\frac{V_{4} \sinh \pi \epsilon}{\sqrt{2 \pi^{2} \alpha^{\prime}}} \int_{0}^{\infty} \frac{d t}{\sqrt{t}} \frac{e^{\frac{-r^{2} t}{2 \pi \alpha^{\prime}}}}{\left(8 \pi^{2} \alpha^{\prime} t\right)^{2}} \tan \pi \epsilon t / 2
$$

Making a velocity expansion, we get

$$
\mathcal{V}_{D 4-D 8}=\frac{V_{4}}{\left(8 \pi^{2} \alpha^{\prime}\right)^{2}} \frac{v^{2}}{2}\left(-\frac{r\left(1-v^{2} / 2\right)}{\pi \alpha^{\prime}}+\frac{v^{2}}{12} \frac{\pi \alpha^{\prime}}{r^{3}}\right)+O\left(v^{6}\right)
$$

The physically relevant quantity is the sum

$$
\mathcal{V}_{D 4-\Omega 8}+16 \mathcal{V}_{D 4-D 8}=\frac{V_{4}}{\left(8 \pi^{2} \alpha^{\prime}\right)^{2}} \frac{v^{4} \pi \alpha^{\prime}}{r^{3}}+O\left(v^{6}\right)
$$

Thus the metric on the moduli space of a D4-brane probe is flat even at short distances. If the D8-branes were not all placed at the orientifold plane there would be a non-trivial moduli space metric. It turns out that this is the same as the linear potential experienced by the D0-brane probe when not all the D8-branes are placed on top of the orientifold plane. It is interesting that the $1 / r^{3}$ deviation from flat metric seen by the D0-brane probe is also the behaviour of the coefficient of the higher dimensional $v^{4}$ term in the D4-brane potential. Thus a probeindependent characterization of an underlying spacetime may exist even at short distances. We also note that the leading terms in the velocity expansion of all the individual potentials calculated in the short distance approximation do not receive corrections from the massive open string states at large distances because the differences of the dimensions of the probes and the targets are always a multiple of four [24]. But the combined potential due to the orientifold plane and D8-branes is such that the leading terms always cancel. The coefficient of the surviving next-to-leading term is no longer an inegral over a modular form of weight zero and thus massive open string states do not decouple from the large distance physics. 


\section{Discussion and concluding remarks}

In this work we have addressed two issues. One relates to the probe dependence of the physics of Type I' background. We have seen that at distances large compared to the string scale, the moduli space actions for both the D0 and D4-branes are consistent with a flat space and constant dilaton background. This probe-independence of large distance physics is what we would have expected from previous works [17, 18] and from physical considerations. At distances shorter than the string scale, we get different moduli space actions for the D0 and D4-branes. In particular, while the D0-brane perceives a nontrivial metric, the metric seen by D4-brane is flat. However, this probe-dependence need not worry us. This is because even if there exists a description of the short distance physics in terms of an underlying spacetime picture in a probe-independent way, the metric, dilaton, etc. may couple differently to different probes, giving rise to different moduli space actions. Thus there is no reason to expect the moduli space metrics to be the same for the two probes. The good news is that the D4-brane does have a nontrivial action. In particular, we have seen that the coefficient of the $v^{4}$ term shows exactly the same dependence on distance from the fixed plane as the coefficient of the $v^{2}$ term in the D0-brane moduli space action. This encourages us to think that even at short distances there might be a universal notion of an underlying spactime physics which is simply perceived differently by different probes.

The other issue that we have addressed is the matrix theory conjecture for M-theory in the contex of Type I' compactification. We have argued that the matrix model of [11, 12, 13] does not reproduce the expected spacetime gravitational physics of the Type $\mathrm{I}^{\prime}$ theory. The massive open string modes neglected in the matrix model do not decouple in the large distance limit. It is not clear to us whether any simple modification ( which changes the number of degrees of freedom by a finite amount) of the matrix model would do the job.

Finally, we mention that all the systems examined so far for which the matrix theory conjecture seems to run into problems [9, 10] have eight supersymmetries as opposed to sixteen in the model for which the original conjecture was madel. Matrix quantum mechanics models with eight supersymmetries were examined in [16] and shown to admit loop corrections to the moduli space metric. In fact, the moduli space metric is largely unconstrained. In this context it is interesting that the discrepancy between the moduli space metrics for large and small black holes in the calculations of Douglas, Polchinski and Strominger [27] also occurs for a system with eight supersymmetries. One possibility is that the discrepancy is genuine and is explained by the fact that massive open string modes do not decouple from large distance physics, just as we have seen happen in the Type $\mathrm{I}^{\prime}$ case. In this scenario the $1 / r^{4}$ term in the moduli space metric, which reflects the existence of a horizon, would be absent for small black holes. But then we must explain why many calculations work for small black holes just as well as for large ones 3 . It is clearly of crucial importance to resolve these issues for further progress in the area of black hole physics in string theory. Another interesting example of a system with eight supersymmetries is M-theory compactified on $T^{5} / Z_{2}$ [28, 29], the matrix model construction

\footnotetext{
${ }^{2}$ See, however, [26] for an example of a quantum mechanics system with eight supersymmetries which does reproduce supergravity results. We thank M. Douglas for pointing out this reference to us.

3 includes a list of references on this subject.
} 
for which has been discussed in [30]. It would be interesting to repeat the present analysis for this background also. Both these problems are currently under investigation.

\section{References}

[1] T. Banks, W. Fischler, S. H. Shenker and L. Susskind, "M Theory as a Matrix Model: A Conjecture," Phys. Rev. D55 (1997) 5112, hep-th/9610043.

[2] P. K. Townsend, "The Eleven-Dimensional Membrane Revisited," Phys. Lett. B350 (1995) 184, hep-th/9501068.

[3] E. Witten, "String Theory in Various Dimensions," Nucl. Phys. B443 (1995) 85, hep-th/9503124.

[4] R. Dijkgraff, E. Verlinde and H. Verlinde, "Matrix String Theory," hep-th/9703030.

[5] J. Polchinski and P. Pouliot, "Membrane Scattering with M Momentum Transfer," hep-th/9704029.

[6] K. Becker and M. Becker, "A Two-Loop Test of M(atrix) Theory," hep-th/9705091.

[7] K. Becker, M. Becker, J. Polchinski and A. Tseytlin, "Higher Order Graviton Scattering in M(atrix) Theory," hep-th/9706072.

[8] T. Banks, "The State of Matrix Theory," hep-th/9706168.

[9] M. R. Douglas, H. Ooguri and S. H. Shenker, "Issues in (M)atrix Model Compactification," Phys. Lett. B402 (1997) 36, hep-th/9702203.

[10] O. J. Ganor, R. Gopakumar and S. Ramgoolam, "Higher Loop Effects in M(atrix) Orbifolds," hep-th/9705188.

[11] U. H. Danielsson and G. Ferretti, "The Heterotic Life of the D-particle," hep-th/9610082.

[12] S. Kachru and E. Silverstein, "On Gauge Bosons in the Matrix Model Approach to M theory," Phys. Lett. B396 (1997) 76, hep-th/9612162.

[13] N. Kim and S. J. Rey, "M(atrix) Theory on an Orbifold and Twisted Membrane," hep-th/9701139.

[14] P. Horava and E. Witten, "Heterotic and Type I String Dynamics from Eleven Dimensions," Nucl. Phys. B460 (1996) 506, hep-th/9510209.

[15] P. Horava and E. Witten, "Eleven Dimensional Supergravity on a Manifold with Boundary," Nucl. Phys. B475 (1996) 94, hep-th/9603142.

[16] T. Banks, N. Seiberg and E. Silverstein, "Zero and One-dimensional Probes with N=8 Superysmmetry," Phys. Lett. B410 (1997) 30, hep-th/9703052. 
[17] J. Polchinski and E. Witten, "Evidence for Heterotic-Type I String Duality," Nucl. Phys. B460 (1996) 525, hep-th/9510169.

[18] N. Seiberg, "Five Dimensional SUSY Field Theories, Non-trivial Fixed Points and String Dynamics," Phys. Lett B388 1996 753, hep-th/9608111.

[19] Y. Cai and J. Polchinski, "Consistency of Open Superstring Theories," Nucl. Phys. B296 (1988) 91.

[20] M. Billo, P. Di Vecchia and D. Cangemi, "Boundary States for Moving D-branes," hep-th/9701190.

[21] O. Bergman, M. R. Gaberdiel and G. Lifschytz, "Branes, Orientifolds and the Creation of Elementary Strings," hep-th/9705130.

[22] J. M. Pierre, "Interactions of Eight-branes in String Theory and M(atrix) Theory," hep-th/9705110.

[23] C. Bachas, "D-Brane Dynamics," Phys. Lett. B374 (1996) 37, hep-th/9511043.

[24] M. R. Douglas, D. Kabat, P. Pouliot and S. H. Shenker, "D-branes and Short Distances in String Theory," Nucl. Phys. B485 (1997) 85, hep-th/9608024.

[25] J. Polchinski, "TASI Lectures on D-branes," hep-th/9611050.

[26] M. Berkooz and M. R. Douglas, "Five-branes in M(atrix) Theory," Phys.Lett. B395 (1997) 196, hep-th/9610236.

[27] M. R. Douglas, J. Polchinski and A. Strominger, "Probing Five-Dimensional Black Holes with D-branes," hep-th/9703031

[28] K. Dasgupta and S. Mukhi, "Orbifolds of M-theory," Nucl. Phys. B465 (1996) 399, hep-th/9512196.

[29] E. Witten, "Five-branes And $M$-Theory On An Orbifold," Nucl.Phys. B463 (1996) 383, hep-th/9512219.

[30] N. Kim and S. J. Rey, "M(atrix) Theory on $T_{5} / Z_{2}$ Orbifold and Five-Branes," hep-th/9705132. 